\title{
Association of sex and atrial fibrillation therapies with patient-reported outcomes
}

\author{
Kelly Therese Gleason (D) , ${ }^{1}$ Cheryl Renee Dennison Himmelfarb, ${ }^{1}$ Daniel Ernest Ford, ${ }^{1}$ \\ Harold Lehmann, ${ }^{1}$ Laura Samuel, ${ }^{1}$ Sandeep Jain, ${ }^{2}$ Gerald Naccarelli, ${ }^{3}$ Vikas Aggarwal, ${ }^{4}$ \\ Saman Nazarian ${ }^{5}$
}

- Additional material is published online only. To view please visit the journal online (http://dx.doi.org/10.1136/ heartjnl-2019-314881)

${ }^{1}$ School of Nursing, Johns Hopkins University, Baltimore, Maryland, USA

${ }^{2}$ Heart and Vascular Institute, University of Pittsburgh Department of Medicine, Pittsburgh, Pennsylvania, USA ${ }^{3}$ Heart and Vascular Institute, Penn State Health Milton S. Hershey Medical Center, Hershey, Pennsylvania, USA ${ }^{4}$ Division of Cardiology, Department of Internal Medicine, University of Michigan Health System, Ann Harbor, Michigan, USA ${ }^{5}$ Perelman School of Medicine, University of Pennsylvania, Philadelphia, Pennsylvania, USA

\section{Correspondence to}

Kelly Therese Gleason, School of Nursing, Johns Hopkins University, Baltimore, MD 21218, USA:

kgleaso2@jhmi.edu

Received 8 February 2019

Revised 2 May 2019

Accepted 5 May 2019

Published Online First

22 May 2019

\section{Linked}

- http://dx.doi.org/10.1136/ heartjn-2019-315288

Check for updates

(c) Author(s) (or their employer(s)) 2019. No commercial re-use. See rights and permissions. Published by BMJ.

To cite: Gleason KT, Dennison Himmelfarb $C R$ Ford DE, et al. Heart 2019:105:1642-1648.

\section{ABSTRACT}

Objectives Women report higher atrial fibrillation (AF) symptom severity and receive less AF therapies than their male counterparts. It is understudied if differences in $\mathrm{AF}$ therapies received explains sex differences in $\mathrm{AF}$ symptom severity. We investigate the impact of sex and AF therapies on patient-reported outcomes.

Methods Participants were recruited $(n=953)$ across four academic medical centres with an AF diagnosis and age $\geq 18$ years. Patient-reported outcomes (AF symptom severity, AF-related quality of life, functional status and emotional status) were determined by biannual surveys. We performed multiple linear regressions on propensitymatched cohorts to determine the association of AF therapies and sex on patient-reported outcomes. Results Our study population ( $n=953)$ was $65 \%$ male $(n=616), 93 \%$ white $(n=890)$ and $72( \pm 10)$ years old. Individuals receiving rate control therapy reported comparatively lower AF-related quality of life $(-7.22$, $95 \% \mathrm{Cl}-11.51$ to -2.92$)$ and poorer functional status $(-3.69,95 \% \mathrm{Cl}-5.27$ to -2.12$)$. Individuals receiving rhythm control strategies did not report significantly different patient-reported outcomes. Women were more likely to report poorer functional status $(-2.63,95 \% \mathrm{Cl}$ -3.86 to -1.40 ) and poorer AF-related quality of life, higher anxiety (2.33, $95 \% \mathrm{Cl} 1.07$ to 3.59$)$, higher symptoms of depression $(1.48,95 \% \mathrm{Cl} 0.31$ to 2.65$)$ and AF symptom severity $(0.29,95 \% \mathrm{Cl} 0.07$ to 0.52$)$.

Conclusions Female sex was associated with comparatively poorer AF symptom severity and quality of life, and this association remained after accounting for AF therapies received. Receiving rate control medication alone was associated with comparatively poorer AFrelated quality of life and functional status.

\section{INTRODUCTION}

Current guidelines recommend that providers make clinical decisions regarding atrial fibrillation (AF) therapies based on a patients' symptoms. ${ }^{1}$ AF guidelines recommend a series of therapies based on continuing report of bothersome symptoms, including: a strict rate control strategy, rhythm control medications, catheter ablation and serial cardioversions. ${ }^{1}$ Understanding how $\mathrm{AF}$ therapies are associated with patient-reported outcomes (PROs) is necessary to guide clinical decision making. AF symptoms have been linked to PROs, including: emotional status (higher anxiety and symptoms of depression), ${ }^{2}{ }^{3}$ lower quality of life $^{4}$ and lower functional status. ${ }^{5}$ Quality of life, emotional status and functional status are invaluable to an individual's health and underscore the importance of managing AF symptoms.

Therapies that target AF symptoms may be underused among women. ${ }^{6}$ Women are less likely to receive rhythm control treatments including cardioversions and ablations that reduce symptom severity. ${ }^{7-9}$ Differences in therapeutic effects by female sex are established in other cardiovascular disease therapies, ${ }^{10}$ and evidence suggests these differences exist in AF: women have comparatively low quality of life and high symptom severity in comparison to their counterparts. ${ }^{711} 12$ Whether differences in therapies received explains the sex differences in PROs, particularly symptoms and quality of life, among individuals with $\mathrm{AF}$ is underexplored. It is essential to understand the interplay of AF therapies and sex on these PROs.

Investigation of differences in key PROs among individuals with $\mathrm{AF}$ is important because sex differences may widen cardiovascular health disparities. ${ }^{13}$ The purpose of this study was to determine the association of AF therapies and sex with subsequent PROs (anxiety, depression, functional status, quality of life and symptom severity).

\section{METHODS}

\section{Design and setting}

The University of Pittsburgh/University of Pittsburgh Medical Center, Penn State College of Medicine, Temple University Hospital and Johns Hopkins University (PaTH) Clinical Data Research Network uses clinical data from electronic medical records (EMRs) combined with PROs. ${ }^{14}$ The PaTH AF is a cohort study at five academic medical centres. ${ }^{14}$ The PaTH AF has two components: (1) a clinical database of over 95302 individuals identified through EMRs; this dataset is thus limited to deidentified information from EMRs, and (2) a cohort of 953 consented individuals who were recruited from the larger clinical database. Patients provided electronic informed consent to: (1) complete questionnaires every 6 months on their PROs and (2) allow researchers to extract data from their EMRs and link these data to their PROs.

\section{Inclusion and exclusion criteria}

Inclusion criteria for the PaTH AF clinical database included: diagnosis of AF by ICD-9-CM (International Classification of Diseases, 9th Revision, Clinical Modication) and ICD-10-CM (International 
Classification of Diseases, 10th Revision, Clinical Modification) codes or electrocardiographic reading, three outpatient, non-emergency and non-inpatient visits since 1 January 2011, age $\geq 18$ years and ability to speak, read and understand English. Individuals were excluded if they received the diagnosis of $\mathrm{AF}$ within a month of cardiac or abdominal surgery, or a thyroid-related diagnosis, and 12 months before or after prescription for methimazole or propylthioracil. All patients meeting the criteria at each institution were in $\mathrm{PaTH}$ the clinical database.

\section{Recruitment, screening and data collection procedures}

Individuals identified in the clinical database were recruited through in-person, email, phone, patient portal messaging and post mail beginning in August 2015 (online supplementary figure A1). ${ }^{15}$ The consented participants were compared on key characteristics to the individuals in the clinical database (online supplementary table A1). Participants recruited through email and post mail completed surveys through REDCap (Vanderbilt University, Tennessee), and participants recruited through the patient portal completed the surveys in MyChart (EPIC software, Wisconsin). All surveys could be completed by the patient online. Participants completed questionnaires on PROs at time of enrolment and every 6 months following enrolment. Healthcare utilisation, procedures, medications, diagnoses and body mass index (BMI) are collected through EMRs. This analysis consists of participants' first 3 PRO surveys (time of enrolment, 6 months and 1 year).

\section{Patient-reported outcomes}

Symptoms and quality of life were measured using the Atrial Fibrillation Effect on QualiTy of Life (AFEQT). ${ }^{16}$ The AFEQT is a disease-specific quality of life instrument consisting of four subscales: symptoms, treatment concerns, daily activities and treatment satisfaction. The subscales for symptoms (four items), treatment concerns (six items) and daily activities (eight items) are added together to create an AF-related quality of life score (18 items). The AFEQT has high internal consistency (Cronbach's $\alpha$ : >0.8) and has been validated in a large, community-based cohort. ${ }^{16}$ In this sample, the AFEQT had a Cronbach's $\alpha$ of 0.93 . The symptoms subscale consists of four items rated on a 7-point Likert scale and has a previously reported Cronbach's $\alpha$ of $0.5^{16}$ and a Cronbach's $\alpha$ of 0.81 in this sample. The symptoms subscale score formed by calculating the mean score of the questions answered. The AFEQT total scale scores range from 0 to 100 , with 0 representing the worst possible quality of life.

Emotional and functional status were measured using the PROMIS (Patient-Reported Outcomes Measurement Information System)-29 emotional status (anxiety and depression) and functional status domains. ${ }^{17}$ The emotional status domain consists of four questions related to anxiety and four questions related to depression rated on a five-point Likert-type scale. The functional status domain consists of four items related to functional limitations rated on a five-point Likert-type scales. High internal consistency (Cronbach's $\alpha$ : $>0.8$ ) for both the emotional and function status domains has been previously reported, ${ }^{17}$ and was similarly found in this sample. The raw scores of the anxiety, depression and functional status domains were converted to a scaled T-score that ranges from 41 to 79.4 for depression, from 40.3 to 81.6 for anxiety and from 22.9 to 56.9 for function using PROMIS scoring guidelines. ${ }^{18}$ The scaled T-score is created based on PROMIS scores' mean of 50 and SD of 10 in a generalised, referent population.

\section{AF therapies and sex}

Rate control (rate control medication), rhythm control (rhythm control medication, cardioversion and/or ablation) and sex were the key independent variables. Each was analysed as a binary variable. Patients were classified as receiving rate control medication or rhythm control medication if they were prescribed a medication in an outpatient setting in the respective category within 1 year prior to enrolment. One year was used as a cut-off point since prescriptions are renewed on an annual basis. Rate and rhythm control medications were categorised based on practice guidelines. ${ }^{1}$ Ablation was classified based on provider billing code CPT 93656 within the past 3 years. Cardioversion was classified based on provider billing codes CPT 92960, 92961 or ICD-9 99.61 within the past 3 years. Three years was used as a cut-off point since ablations and cardioversions are more commonly used as a one-time procedure to correct rhythm or limit symptom severity. ${ }^{1}$ Sex was determined through EMR documentation.

\section{Other individual and health characteristics}

Other independent variables were assessed as potential confounders. Data for age, education (>college (ref.), college, some college and $\leq$ high school) and race (white (ref.), black and other) were collected in the baseline questionnaire. Comorbidities and BMI (normal weight (ref.), underweight, overweight and obese) were determined through EMR data. Comorbidities were measured using the grouped Charlson comorbidities index score, which is calculated using the Deyo version. ${ }^{19}$ The grouped Charlson comorbidities index score rates comorbidities on a $0-2$ scale. Dummy variables for study site were used.

\section{Statistical methods}

We used the statistical software STATA V.15.0 for all analyses. Patterns of missing data were assessed for each variable included in the models and, for variables with greater than $5 \%$ missing, missing data were imputed using multiple imputation. ${ }^{20}$ We created 100 imputations for each PRO since $13 \%$ of these measures were missing. When we compared results using completed cases $(n=829)$ only against results using multiple imputation $(\mathrm{n}=953)$, we found that inferences did not differ across the models. We conducted a power analysis with an alpha of 0.05 and determined that we had power $>0.80$, thus attention to effect sizes associated with significant results was important.

Propensity-score matching was used to address potential confounding of the association between AF therapies and PROs. Nearest neighbour matching $(1: 10)$ with replacement was conducted separately for individuals solely on rate control medications and individuals on rhythm control strategies. Analyses were restricted to individuals who could be matched to another participant with a similar probability (calliper $\leq 0.04$ ) of receiving the therapy to reduce the possibility of model extrapolations and the biasing effects of model misspecification. ${ }^{21}$ The individuals on rhythm control strategy $(\mathrm{n}=325)$ were matched to 525 'control' individuals, and individuals on rate control medication only $(n=144)$ were matched to 708 'control' individuals. The propensity score models for rate control and rhythm control strategies reduced bias on key variables (online table A2). Hypothesised associations between rate control and rhythm control strategies and each PRO were tested separately using linear regression models among propensity score matched subsamples. All models adjusted for individual characteristics (sex, age and education level) and health characteristics (BMI 
and comorbidities). Regression coefficients were not standardised in order for the PROs to be interpreted in their unit of measurement.

A subset of individuals underwent a rhythm control strategy including ablation, cardioversion or rhythm control medication $(n=111)$ or only a rate control medication $(n=26)$ after completing their baseline PROs survey, which allowed for comparisons in PROs change following therapy. We analysed the mean change in symptoms of AF, depression and anxiety, and functional status in bivariate regression models to determine the effect of rhythm and rate control medication for this subset of individuals.

\section{RESULTS \\ Sample characteristics}

Table 1 depicts demographic data specific to participants stratified by recent rate and rhythm control therapies. Our study population $(n=953)$ were $65 \%$ male $(n=616), 93 \%$ white $(n=890)$ and $72( \pm 10)$ years old on average. Thirty-seven per cent of participants had less than a college degree $(n=330)$. A comorbidity index of 2 was present in 39\% $(n=369)$ of the sample. The common comorbidities were hypertension (71\%), heart failure $(28 \%)$ and diabetes $(22 \%)$. The majority of the population was overweight $(35 \%)$ or obese $(44 \%)$. History of ablation was present in $7 \%(n=70), 21 \%$ had a cardioversion $(n=190), 56 \%$ had a history of being on rate control medication $(n=538)$ and $58 \%$ had a history of being on rhythm control medication $(\mathrm{n}=554)$. Rhythm control medication had been prescribed to $19 \%(n=182)$ of the sample in the past year, and rate control had been prescribed to $16 \%(n=158)$ of the sample in the past year.

Our sample reported poorer average functional status (47.3), and lower symptoms of anxiety (48.9) and depression (46.9) than the general population mean. Participants reported an average of 2.21 on the AF symptoms subscale, which can be interpreted as the participants were on average 'a little bothered' by their AF symptoms. On average, the AFEQT total score was 62.72 on a scale of $0-100$, with 0 being the lowest AF-related quality of life.

\section{Rate control strategies matched sample}

Among the sample matched on rate control medication prescription, rate control medication was associated with significantly poorer functional status $(-3.69,95 \% \mathrm{CI}-5.27$ to -2.12$)$ and AF-related quality of life $(-7.22,95 \% \mathrm{CI}-11.51$ to -2.92$)$ (table 2, figure 1). Rate control medications were not associated with anxiety, AF symptoms and depression. Among the subset of individuals $(n=25)$ who had a documented rate control medication prescription only after the baseline PROs, the mean change in PROs was not significantly different from individuals with no documented rate or rhythm control strategies (see online appendix table A4).

Women were more likely to report poorer functional status, higher symptoms of anxiety, depression and AF and AF-related quality of life. Older age was associated with poorer functional status, lower symptoms of anxiety, depression and AF symptoms and high AF-related quality of life. Compared with individuals with greater than a college education, individuals with less education reported poorer functional status. Individuals with some college reported higher symptoms of AF and anxiety and poor AF-related quality of life compared with individuals with greater than a college education.

\section{Rhythm control strategies matched sample}

Among the sample matched on history of rhythm control strategies, rhythm control strategies were not associated with PROs (table 3$)$. Among the subset of individuals $(n=111)$ who had a documented rhythm control medication prescription only after the baseline PROs, the mean change in PROs was not significantly different from individuals with no documented rate or rhythm control strategies (see online appendix).

Women reported higher symptoms of AF, anxiety and depression and poorer functional status and AF-related quality of life. Individuals with a college education, some college and less than college had poor functional status compared with individuals with greater than a college education. Individuals with some college education had higher symptoms of AF and anxiety and poorer AF-related quality of life compared with individuals with greater than a college education. Older age was associated with poorer functional status, lower symptoms of anxiety, depression and AF and higher AF-related quality of life.

\section{DISCUSSION}

In this multi-institutional cohort of 953 individuals with $\mathrm{AF}$ propensity score matched on AF therapies, AF therapies were not associated with more favourable PROs, and sex was more strongly associated with PROs than AF therapies. Rate control strategies were associated with poorer functional status and AF-related quality of life. Women reported comparatively poorer functional status and higher symptoms of anxiety, depression and AF compared with their male counterparts.

The lack of a positive association between AF therapies and more favourable PROs was unexpected based on prior studies' findings. ${ }^{223}$ We cannot determine a causal relationship and therapeutic effectiveness due to the design of this cohort study. Since guidelines recommend symptomatic individuals receive rhythm control therapies, those receiving these therapies may have had poorer PROs at baseline. The side effects of rate control medications may lead to comparatively poor PROs, particularly fatigue, ${ }^{24}$ though this study did not explicitly capture side effects. The individuals in the study have chronic conditions including heart failure that also affects quality of life, functional status and symptoms.

Our previous study in this cohort found that women were more likely to report AF symptoms and poorer quality of life than men. ${ }^{25}$ We conducted this study to build on this finding by exploring if sex differences in rhythm and rate control therapies received accounts for the higher symptom severity experienced by women, since women are less likely to receive rhythm control treatments including cardioversions and ablations that reduce symptom severity. ${ }^{7-9}$ We found that sex differences in AF symptoms severity and quality of life persisted after we accounted for rate and rhythm therapies received by creating matched samples. This finding aligns with evidence suggesting that ablation is as effective in women as in men. ${ }^{26} 27$ Thus, sex differences in AF therapies received do not appear to explain sex differences in AF symptom severity and quality of life. Women have a higher resting heart rate than men and differences in electrophysiology by sex have been established; however, whether there are pathophysiological differences in AF experience by sex is overall understudied. $^{28}$

Female sex and other individual characteristics (age and low education level) associated with high AF symptoms were also associated with high symptoms of anxiety and depression. Higher symptoms of anxiety and depression may contribute to higher AF symptom severity. ${ }^{3}$ Individuals with high levels of psychological 
Table 1 Sample demographic characteristics by rate or rhythm control therapy, $n=953$

\begin{tabular}{|c|c|c|c|c|}
\hline & Total & No recent therapy & $\begin{array}{l}\text { Recent rate or rhythm } \\
\text { control therapy }\end{array}$ & \\
\hline & $\%(n)$ & -550 & -403 & OR/ $\beta(95 \% \mathrm{Cl})^{*}$ \\
\hline \multicolumn{5}{|l|}{ Sex } \\
\hline Female & 35 (337) & 35 (194) & 35 (143) & 1.01 (0.77 to 1.32$)$ \\
\hline Male & $65(616)$ & 65 (356) & $65(260)$ & \\
\hline \multicolumn{5}{|l|}{ Race } \\
\hline White & $93(890)$ & $94(514)$ & $94(376)$ & Reference \\
\hline Black & $5(47)$ & $5(29)$ & $4(18)$ & 0.88 (048 to 1.61$)$ \\
\hline Other & $1(16)$ & $1(7)$ & $2(9)$ & $1.17(0.39$ to 3.51$)$ \\
\hline Age & 71.7 (SD 10) & 72.47 (SD 10.43) & 70.51 (SD 9.88) & $0.98(0.97$ to 0.99$)$ \\
\hline \multicolumn{5}{|l|}{ Education } \\
\hline$>$ Than college & 40 (358) & 38 (199) & 44 (164) & Reference \\
\hline College & 22 (198) & 24 (124) & $20(75)$ & $0.73(0.52$ to 1.04$)$ \\
\hline Some college & $23(200)$ & $24(123)$ & $21(79)$ & $0.78(0.55$ to 1.11$)$ \\
\hline High school or less & $14(130)$ & $14(74)$ & $15(58)$ & 0.95 (0.64 to 1.42$)$ \\
\hline \multicolumn{5}{|c|}{ Grouped comorbidity (Charlson) index } \\
\hline \multicolumn{5}{|l|}{0} \\
\hline 1 & 40 (374) & $40(224)$ & $39(152)$ & Reference \\
\hline \multirow[t]{2}{*}{2} & $21(195)$ & $21(118)$ & $21(82)$ & 1.02 (0.72 to 1.45$)$ \\
\hline & $39(369)$ & $39(214)$ & 40 (159) & $1.09(0.82$ to 1.46$)$ \\
\hline \multicolumn{5}{|l|}{ BMI } \\
\hline Normal $18.5-24.9 \mathrm{~kg} / \mathrm{m}^{2}$ & $21(193)$ & $18(97)$ & $25(101)$ & Ref \\
\hline Overweight $\left(25-<30 \mathrm{~kg} / \mathrm{m}^{2}\right)$ & $35(324)$ & $40(223)$ & $32(128)$ & 0.67 (0.46 to 0.96$)$ \\
\hline Obese $\left(\geq 30 \mathrm{~kg} / \mathrm{m}^{2}\right)$ & $44(408)$ & $42(233)$ & $43(175)$ & $0.85(0.60$ to 1.20$)$ \\
\hline \multicolumn{5}{|l|}{ Ablation } \\
\hline Yes & $7(70)$ & & & \\
\hline No & $93(883)$ & & & \\
\hline \multicolumn{5}{|l|}{ Cardioversion } \\
\hline Yes & $21(190)$ & & & \\
\hline No & $79(763)$ & & & \\
\hline \multicolumn{5}{|l|}{ Rate control medication } \\
\hline \multicolumn{5}{|l|}{ History of prescription } \\
\hline \multicolumn{5}{|l|}{ Yes } \\
\hline No & $52(492)$ & & & \\
\hline Prescription in the past year & $48(461)$ & & & \\
\hline \multicolumn{5}{|l|}{ Yes } \\
\hline \multicolumn{5}{|l|}{ No } \\
\hline & $16(158)$ & & & \\
\hline & $83(799)$ & & & \\
\hline \multicolumn{5}{|l|}{ Rhythm control medication } \\
\hline \multicolumn{5}{|l|}{ History of prescription } \\
\hline \multicolumn{5}{|l|}{ Yes } \\
\hline No & $54(514)$ & & & \\
\hline Prescription in the past year & $46(439)$ & & & \\
\hline \multicolumn{5}{|l|}{ Yes } \\
\hline \multicolumn{5}{|l|}{ No } \\
\hline & $28(267)$ & & & \\
\hline & 72 (686) & & & \\
\hline Quality of life & $62.72(25.80)$ & $63.84(25.63)$ & $60.84(26.03)$ & $-2.04(-5.91$ to 1.83$)$ \\
\hline Symptoms & $2.21(1.4)$ & $2.16(1.35)$ & $2.30(1.50)$ & $0.13(-0.08$ to 0.34$)$ \\
\hline Functional status & $47.30(8.8)$ & $47.19(8.92)$ & $47.46(8.61)$ & $0.27(-0.93$ to 1.47$)$ \\
\hline Anxiety & $48.93(8.75)$ & $49.09(9.07)$ & $48.75(8.28)$ & $-0.34(-1.53$ to 0.85$)$ \\
\hline Depression & $46.86(8.08)$ & $46.98(8.52)$ & $46.79(7.86)$ & $-0.19(-1.29$ to 0.92$)$ \\
\hline
\end{tabular}

* Results indicate the influence of each variable on being prescribed recent rate or rhythm control therapy in unadjusted models.

BMI, body mass index. 
Table 2 Rate control strategies and patient-reported outcomes in propensity-score matched cohorts

\begin{tabular}{|c|c|c|c|c|c|}
\hline & Function & Anxiety & Depression & Symptoms & AF-related QOL \\
\hline & $(\beta, 95 \% \mathrm{Cl})$ & $(\beta, 95 \% \mathrm{Cl})$ & $(\beta, 95 \% \mathrm{Cl})$ & $(\beta, 95 \% \mathrm{Cl})$ & $(\beta, 95 \% \mathrm{Cl})$ \\
\hline Rate control strategy & $-3.69(-5.27$ to 2.12$)$ & $1.35(-0.29$ to 2.98$)$ & $1.50(-0.03$ to 3.03$)$ & $0.05(-0.22$ to 0.33$)$ & $-7.22(-11.51$ to 2.92$)$ \\
\hline Sex & $-2.63(-3.86$ to 1.40$)$ & 2.33 (1.07 to 3.59$)$ & $1.48(0.31$ to 2.65$)$ & $0.29(0.07$ to 0.52$)$ & $-5.04(-8.51$ to 1.57$)$ \\
\hline Age & $-0.19(-0.25$ to 0.13$)$ & $-0.15(-0.20$ to 0.08$)$ & $-0.12(-0.17$ to 0.07$)$ & $-0.02(-0.04$ to 0.01$)$ & $0.14(-0.020 .31)$ \\
\hline \multicolumn{6}{|l|}{ Education level } \\
\hline$>$ Than college & Ref & Ref & Ref & Ref & Ref \\
\hline College & $-1.79(-3.32$ to 0.26$)$ & $-0.04(-1.60$ to 1.52$)$ & $0.19(-1.27$ to 1.66$)$ & $0.01(-0.26$ to 0.28$)$ & $-0.76(-4.99$ to 3.46$)$ \\
\hline Some college & $-1.84(-3.38$ to 0.30$)$ & $1.79(0.21$ to 3.37$)$ & $1.19(-0.28$ to 2.65$)$ & $0.40(0.13$ to 0.68$)$ & $-5.07(-9.28$ to 0.87$)$ \\
\hline High school or less & $-1.92(-3.72$ to 0.12$)$ & $-0.16(-1.98$ to 1.67$)$ & $0.29(-1.41$ to 1.99$)$ & $0.04(-0.27$ to 0.36$)$ & $-0.86(-5.74$ to 4.02$)$ \\
\hline \multicolumn{6}{|l|}{ Comorbidity index } \\
\hline 0 & Ref & Ref & Ref & Ref & Ref \\
\hline 1 & -0.96 ( -2.50 to 0.58$)$ & $0.64(-0.94$ to 2.22$)$ & $1.27(-0.21$ to 2.74$)$ & $-0.07(-0.34$ to 0.20$)$ & $-0.96(-5.20$ to 3.28$)$ \\
\hline 2 & $-2.28(-3.59$ to 0.97$)$ & $0.79(-0.55$ to 2.12$)$ & 1.44 (0.18 to 2.69$)$ & $0.10(-0.13,0.34)$ & $-3.84(-7.68$ to 0.01$)$ \\
\hline \multicolumn{6}{|l|}{ BMI } \\
\hline Normal & Ref & Ref & Ref & Ref & Ref \\
\hline Overweight & $0.33(-1.30$ to 1.97$)$ & $0.82(-0.83$ to 2.48$)$ & $-0.03(-1.56$ to 1.50$)$ & $-0.05(-0.34$ to 0.23$)$ & -1.21 ( -5.66 to 3.23$)$ \\
\hline Obese & $-3.02(-4.62$ to 1.41$)$ & $0.76(-0.87$ to 2.38$)$ & $0.20(-1.32$ to 1.71$)$ & $0.11(0.13$ to 0.35$)$ & $-6.28(-10.93$, to 1.98$)$ \\
\hline
\end{tabular}

$\mathrm{AF}$, atrial fibrillation; BMI, body mass index; QOL, quality of life.

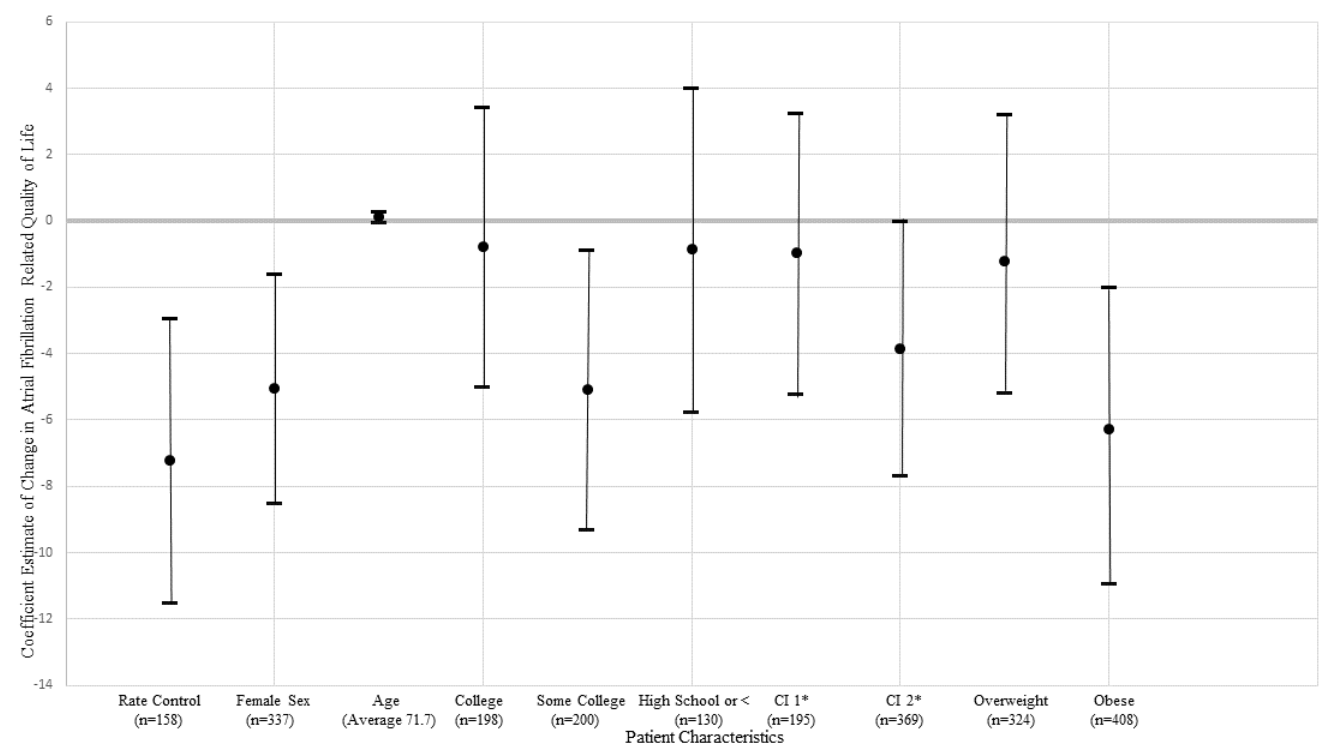

Figure 1 The association of therapies and key patient characteristics with atrial fibrillation-related quality of life in propensity score matched adjust models. Notes: results presented as $\beta, 95 \% \mathrm{Cl}$. The reference groups for sex, education level, comorbidity index and weight were male sex, greater than college, comorbidity index of 0 and normal weight. *Comorbidity index.

distress prior to diagnosis of AF may be more likely to experience symptoms. ${ }^{3} 29$ The association between psychological distress and AF symptoms may be bidirectional: psychological distress may precipitate a symptomatic AF episode, and the symptoms of AF may result in high levels of psychological distress. ${ }^{3}$ Studies specifically designed to examine psychological distress and AF symptoms are needed to draw definitive conclusions.

\section{Clinical implications}

AF symptoms are a chief driver of clinical decision making for AF management. ${ }^{1}$ AF symptoms, emotional status, quality of life and functional status are all linked to each other. ${ }^{2} 3522$ Yet in this study, we found that AF therapies received were not associated with more favourable PROs. Women in this study reported higher AF symptom severity, and they also reported higher anxiety and depression and poorer functional status. Identifying and addressing root causes for individuals' higher anxiety and depression and poorer functional status may improve AF-related PROs. Risk factors for AF, including hypertension, diabetes, obstructive sleep apnoea, obesity, poor diet, smoking and alcohol use, ${ }^{30}$ may contribute to comparatively poorer PROs. Managing these conditions as part of a comprehensive treatment approach may improve functional and emotional status and quality of life, which may, in turn, improve AF symptoms.

\section{Limitations}

There are important limitations to this study that must be acknowledged. The differences in PROs are minimally clinically significant: AFEQT requires a 12-point change for a clinically significant change ${ }^{16}$ and PROMIS similarly requires a change by at least five points for clinical significance. ${ }^{18}$ Recruitment was based on EMR documentation of AF, and thus, biases exist in patient selection. Men, people with fewer comorbidities and younger adults were more likely to join this study. Both this 
Table 3 Rhythm control strategies and patient-reported outcomes in propensity score matched cohorts

\begin{tabular}{|c|c|c|c|c|c|}
\hline & Function & Anxiety & Depression & Symptoms & AF-related QOL \\
\hline & $(\beta, 95 \% \mathrm{Cl})$ & $(\beta, 95 \% \mathrm{Cl})$ & $(\beta, 95 \% \mathrm{Cl})$ & $(\beta, 95 \% \mathrm{Cl})$ & $(\beta, 95 \% \mathrm{Cl})$ \\
\hline Rhythm control strategy* & $0.20(-0.99$ to 1.40$)$ & $-0.44(-1.66$ to 0.78$)$ & $-0.39(-1.53$ to 0.76$)$ & 0.00 (-21 to 0.22$)$ & $-3.23(-6.78$ to 0.32$)$ \\
\hline Sex & $-2.33(-3.57$ to 1.08$)$ & $2.25(0.99$ to 3.50$)$ & $1.51(0.32$ to 2.70$)$ & $0.28(0.05$ to 0.50$)$ & $-4.64(-8.28$ to 1.01$)$ \\
\hline Age & $-0.19(-0.25$ to 0.13$)$ & $-0.14(-0.20$ to 0.08$)$ & $-0.12(-0.17$ to 0.06$)$ & $-0.03(-0.04$ to 0.02$)$ & $0.18(0.01$ to 0.36$)$ \\
\hline \multicolumn{6}{|l|}{ Education level } \\
\hline$>$ Than college & Ref & Ref & Ref & Ref & Ref \\
\hline College & $-1.95(-3.49$ to 0.42$)$ & $-0.10(-1.67$ to 1.46$)$ & $0.31(-1.16$ to 1.77$)$ & $0.04(-0.23$ to 0.31$)$ & $-0.42(-4.93$ to 4.09$)$ \\
\hline Some college & $-1.76(-3.31$ to 0.20$)$ & $1.51(-0.07$ to 3.08$)$ & $1.15(-0.33$ to 2.63$)$ & $0.42(0.14$ to 0.70$)$ & $-6.11(-10.58$ to 11.65 \\
\hline High school or less & $-2.46(-4.26$ to 0.66$)$ & $-0.04(-1.87$ to 1.79$)$ & $0.52(-1.20$ to 2.24$)$ & $0.13(-0.18$ to 0.43$)$ & $-3.09(-8.09$ to 1.90$)$ \\
\hline \multicolumn{6}{|l|}{ Comorbidity index } \\
\hline 0 & Ref & Ref & Ref & Ref & Ref \\
\hline 1 & $-0.76(-2.31$ to 0.79$)$ & 0.93 (-0.65 to 2.51$)$ & $1.17(-0.30$ to 2.64$)$ & $-0.26(-1.37$ to 0.85$)$ & $-0.82(-5.32$ to 3.68$)$ \\
\hline 2 & $-2.47(-3.79$ to 1.15$)$ & 0.76 ( -0.59 to 2.11$)$ & 1.60 (0.34 to 2.86$)$ & $-0.01(-1.11$ to 1.08$)$ & $-5.89(-9.81$ to 1.97$)$ \\
\hline \multicolumn{6}{|l|}{ BMI } \\
\hline Normal & Ref & Ref & Ref & Ref & Ref \\
\hline Overweight & $0.70(-0.94$ to 2.34$)$ & $0.38(-1.29$ to 2.05$)$ & $-0.38(-1.95$ to 1.18$)$ & $-0.12(-0.0$ to 0.17$)$ & $-0.18(-5.05$ to 4.68$)$ \\
\hline Obese & $-2.76(-4.37$ to 1.16$)$ & 0.76 (-0.89 to 2.42$)$ & $0.03(-1.50$ to 1.56$)$ & $0.12(0.26$ to 0.30$)$ & $-6.18(-10.93$ to 1.43$)$ \\
\hline
\end{tabular}

* Rhythm control strategy included cardioversion, ablation and rhythm control medications.

$\mathrm{BMI}$, body mass index; QOL, quality of life.

study sample and the larger PaTH AF clinical database individuals were majority white, which may be reflective of the population in treatment for AF. There is a lower prevalence of this disease among black and Hispanic populations that adds to the challenge of enrolling a racially diverse group of patients.

Relying on EMR data for information on therapies is a significant weakness. Whether the therapies were successful (ie, if rate and rhythm were controlled) is not captured. EMR data are subject to biases that exist from institution to institution and provider to provider and are limited to the care the individuals

\section{Key messages}

\section{What is already known on this subject?}

- Women experience disproportionately poorer atrial fibrillation outcomes, including higher symptom severity and lower quality of life.

- Therapies that target atrial fibrillation symptoms may be underused in women. For example, women with atrial fibrillation are less likely to receive rhythm control treatments including cardioversions and ablations that reduce symptom severity.

\section{What might this study add?}

- Female sex was associated with comparatively poor patientreported outcomes, and this difference persisted after accounting for therapies received.

- Rate control strategies used in isolation (without a rhythm control strategy) were associated with poorer functional status and AF-related quality of life.

\section{How might this impact on clinical practice?}

- The significant influence of female sex on patient-reported outcomes among individuals with atrial fibrillation highlights the importance taking these factors into consideration in clinical decision making.

- Patients initiated on a rate control strategy, alone, may benefit from being monitored to determine if adjusting the therapeutic regimen is necessary to improve quality of life. received at the institutions involved in the study. We chose conservative time windows for therapies in recognition of EMR data limitations. We conducted sensitivity analyses with alternate time windows for medications (history of prescription vs limiting to prescriptions 1 year prior) and ablations (limited to a 1 -year window vs a 3 -year window), and our findings were unchanged. Agreement between patient-reported medication use and documented prescription of medication was assessed in a subset of patients, and high agreement was found using medications prescribed in the past year $(\kappa>0.80)$.

\section{CONCLUSION}

In this study, we found that rate and rhythm control strategies were not significantly associated with more favourable AF-related quality of life and symptom status. Women reported comparatively poorer AF symptom severity, quality of life and functional status and higher symptoms of depression and anxiety, and this finding persisted after accounting for rate and rhythm control strategies. The significant influence of sex and other individual characteristics on PROs, specifically AF-related quality of life, symptom severity, functional status, symptoms of depression and anxiety, among individuals with AF highlights the importance taking these factors into consideration in clinical decision making. Individuals solely receiving a rate control strategy may benefit from closely following AF-related quality of life to ensure adequate management.

Acknowledgements We are grateful to Megan Gauvey-Kern, the project manager at the Johns Hopkins site, for her tremendous work in recruiting and managing the atrial fibrillation cohort, and the PROMIS group, led by Dr Albert Wu, for advising on analyses with PROMIS measures. We would also like to acknowledge Dr Nancy Perrin for providing advice on the statistical analysis and interpretation.

Contributors KG originated the concept of the manuscript and led the writing of the manuscript. $\mathrm{HL}$ and $\mathrm{LS}$ advised on the statistical analyses and contributed to the interpretation of the findings of the manuscript. $\mathrm{HL}$ advised and contributed to the data management and quality. DEF and CRDH advised and contributed to the design and interpretation of the findings. SN, SJ, GN and VA advised and contributed to the design of the analyses and interpretation of the findings as expert cardiologists. All authors have read and approved the manuscript.

Funding KTG received support from predoctoral fellowship in Interdisciplinary Training in Cardiovascular Health Research, T32 NR012704, and Predoctoral Clinical Research Training Program, TL1 TR001078. This publication was funded through a 
Patient-Centered Outcomes Research Institute (PCORI) Award (PCORI CDRN \#130604912) for development of the National Patient-Centered Clinical Research Network, known as PCORnet.

Competing interests SN is a scientific advisor to Biosense Webster, Siemens, Imricor and CardioSolv Inc and principal investigator for research funding to the University of Pennsylvania from Biosense Webster, Siemens and Imricor Inc. SJ receives research support from Medtronic and is a principal investigator for research funding to the University of Pittsburgh from Medtronic, Boston Scientific and St. Jude Medical. GN receives research support from Janssen and serves as a scientific advisor to Janssen, Milestone, Omeicos, Glaxo-Smith-Kline and Aceion.

Patient consent for publication Not required.

Ethics approval The University of Pittsburgh/University of Pittsburgh Medical Center, Penn State College of Medicine, Temple University Hospital and Johns Hopkins University AF cohort study was approved by the Institutional Review Board (JHU IRB00064600).

Provenance and peer review Not commissioned; externally peer reviewed.

ORCID iD

Kelly Therese Gleason http://orcid.org/0000-0002-4203-6517

\section{REFERENCES}

1 January CT. AHA/ACC/HRS Guideline for the Management of Patients With Atrial Fibrillation: Executive Summary. Circulation 2014;2014.

2 McCabe PJ, Schumacher K, Barnason SA. Living with atrial fibrillation: a qualitative study. J Cardiovasc Nurs 2011;26:336-44.

3 Thompson TS, Barksdale DJ, Sears SF, et al. The effect of anxiety and depression on symptoms attributed to atrial fibrillation. Pacing Clin Electrophysiol 2014;37:439-46.

$4 \mathrm{Ha} \mathrm{ACT}$. Health-related quality of life in patients with atrial fibrillation treated with rhythm control versus rate control: Insights from a prospective international registry (registry on cardiac rhythm disorders assessing the control of atrial fibrillation: REC. Circ. Cardiovasc. Qual. Outcomes 2014.

5 Chung MK, Shemanski L, Sherman DG, et al. Functional status in rate- versus rhythmcontrol strategies for atrial fibrillation: results of the Atrial Fibrillation Follow-Up Investigation of Rhythm Management (AFFIRM) functional status substudy. J Am Coll Cardiol 2005;46.

6 Lane DA, Aguinaga L, Blomström-Lundqvist C, et al. Cardiac tachyarrhythmias and patient values and preferences for their management: the European Heart Rhythm Association (EHRA) consensus document endorsed by the Heart Rhythm Society (HRS), Asia Pacific Heart Rhythm Society (APHRS), and Sociedad Latinoamericana de Estimulación Cardíaca y Electrofisiología (SOLEACE). Europace 2015;17:1747-69.

7 Schnabel RB, Pecen L, Ojeda FM, et al. Gender differences in clinical presentation and 1-year outcomes in atrial fibrillation. Heart 2017;103:1024-30.

8 Dagres N, Nieuwlaat R, Vardas PE, et al. Gender-related differences in presentation, treatment, and outcome of patients with atrial fibrillation in Europe: a report from the Euro Heart Survey on Atrial Fibrillation. J Am Coll Cardiol 2007;49:572-7.

9 Lip GY, Laroche C, Boriani G, et al. Sex-related differences in presentation, treatment, and outcome of patients with atrial fibrillation in Europe: a report from the Euro Observational Research Programme Pilot survey on Atrial Fibrillation. Europace 2015;17:24-31.

10 Havranek EP, Mujahid MS, Barr DA, et al. Social Determinants of Risk and Outcomes for Cardiovascular Disease: A Scientific Statement From the American Heart Association. Circulation 2015;132:873-98.
11 Reynolds MR, Lavelle T, Essebag V, et al. Influence of age, sex, and atrial fibrillation recurrence on quality of life outcomes in a population of patients with new-onset atrial fibrillation: the Fibrillation Registry Assessing Costs, Therapies, Adverse events and Lifestyle (FRACTAL) study. Am Heart J 2006;152:1097-103.

12 Goli NM, et al. Educational attainment is associated with atrial fibrillation symptom severity. PACE - Pacing Clin. Electrophysiol 2012

13 Bhave PD, Lu X, Girotra S, et al. Race- and sex-related differences in care for patients newly diagnosed with atrial fibrillation. Heart Rhythm 2015;12:1406-12.

14 Amin W, Tsui FR, Borromeo C, et al. PaTH: towards a learning health system in the Mid-Atlantic region. J Am Med Inform Assoc 2014;21:633-6.

15 Gleason KT, Ford DE, Gumas D, et al. Development and preliminary evaluation of a patient portal messaging for research recruitment service. J Clin Trans/ Sci 2018;2:53-6.

16 Spertus J, Dorian P, Bubien R, et al. Development and validation of the Atrial Fibrillation Effect on QualiTy-of-Life (AFEQT) Questionnaire in patients with atrial fibrillation. Circ Arrhythm Electrophysiol 2011;4:15-25.

17 Cella D, Riley W, Stone A, et al. The Patient-Reported Outcomes Measurement Information System (PROMIS) developed and tested its first wave of adult self-reported health outcome item banks: 2005-2008. J Clin Epidemiol 2010;63:1179-94.

18 Pilkonis PA, Choi SW, Reise SP, et al. Item banks for measuring emotional distress from the Patient-Reported Outcomes Measurement Information System (PROMIS $®$ ): depression, anxiety, and anger. Assessment 2011;18:263-83.

19 Deyo RA, Cherkin DC, Ciol MA. Adapting a clinical comorbidity index for use with ICD-9-CM administrative databases. J Clin Epidemiol 1992;45:613-9.

20 Bounthavong M, Watanabe JH, Sullivan KM. Approach to addressing missing data for electronic medical records and pharmacy claims data research. Pharmacotherapy 2015;35:380-7.

21 Rubin DB, Thomas N. Combining propensity score matching with additional adjustments for prognostic covariates. J Am Stat Assoc 2000;95:573-85.

$22 \mathrm{Ha} \mathrm{AC}$, Breithardt G, Camm AJ, et al. Health-related quality of life in patients with atrial fibrillation treated with rhythm control versus rate control: insights from a prospective international registry (Registry on Cardiac Rhythm Disorders Assessing the Control of Atrial Fibrillation: RECORD-AF). Circ Cardiovasc Qual Outcomes 2014; 7:896-904.

23 Fichtner S, Deisenhofer I, Kindsmüller S, et al. Prospective assessment of short- and long-term quality of life after ablation for atrial fibrillation. J Cardiovasc Electrophysiol 2012;23:121-7.

24 Ko DT, Hebert PR, Coffey CS, et al. Beta-blocker therapy and symptoms of depression, fatigue, and sexual dysfunction. JAMA 2002;288:351.

25 Gleason KT, Dennison Himmelfarb CR, Ford DE, et al. Association of sex, age and education level with patient reported outcomes in atrial fibrillation. BMC Cardiovasc Disord 2019;19:85.

26 Forleo GB, Tondo C, De Luca L, et al. Gender-related differences in catheter ablation of atrial fibrillation. Europace 2007;9:613-20.

27 Winkle RA, Mead RH, Engel G, et al. Long-term results of atrial fibrillation ablation: the importance of all initial ablation failures undergoing a repeat ablation. Am Heart $\lrcorner$ 2011;162:193-200.

28 Kurokawa J, Kodama M, Clancy CE, et al. Sex hormonal regulation of cardiac ion channels in drug-induced QT syndromes. Pharmacol Ther 2016;168:23-8.

29 Schnabel RB, Michal M, Wilde $S$, et al. Depression in atrial fibrillation in the general population. PLoS One 2013;8:e79109.

30 Kirchhof P. ESC guidelines for the management of atrial fibrillation developed in collaboration with EACTS. Russian Journal of Cardiology 2016. 